\title{
Rendimento do tomate cereja em função do cacho floral e da concentra- ção de nutrientes em hidroponia
}

\author{
Marcelo de Q Rocha; Roberta MN Peil; Clarissa M Cogo
}

UFPel-FAEM, Progr. Pós-graduação, C. Postal 354,96010-900 Pelotas-RS; mqrocha@yahoo.com.br; rmpeil@ufpel.tche.br; cissacogo@yahoo.com.br

\section{RESUMO}

Neste trabalho avaliou-se os componentes do rendimento (número e peso médio de frutos por planta) do tomate cereja cultivado em sistema hidropônico, no ciclo verão-outono de 2008, observando a influência da posição do cacho floral e da concentração de nutrientes da solução nutritiva. Foram estudadas quatro concentrações iônicas da solução nutritiva (condutividade elétrica inicial (CE)): 1,3; 1,8; 2,3 e 2,8 dS m${ }^{-1}$. Observou-se que não houve interação significativa entre os fatores concentração de nutrientes da solução nutritiva e posição do cacho floral para todos os componentes de rendimento avaliados. Em relação ao efeito da posição do cacho floral, verificouse que somente para as variáveis índice de pegamento e produção de frutos, estes apresentaram influência significativa nas respostas observadas. A concentração iônica da solução nutritiva apresentou efeito significativo para as variáveis de rendimento peso médio do fruto e produção de frutos por planta. Em termos de peso médio de frutos, as CEs de 1,8 e 2,3 $\mathrm{dS} \mathrm{m}^{-1}$ mostraram-se superiores à $\mathrm{CE}$ de $2,8 \mathrm{dS} \mathrm{m}^{-1}$ e similares à $\mathrm{CE}$ de $1,3 \mathrm{dS} \mathrm{m}^{-1}$, obtendo-se 13,$0 ; 12,5 ; 9,5$ e $10,9 \mathrm{~g}$ por fruto, respectivamente. A produção média de frutos das plantas cultivadas na solução nutritiva com CE de 1,8 dS m ${ }^{-1}$ (296,5 g cacho $^{-1} \mathrm{e} 2,1 \mathrm{~kg}_{\text {planta }}{ }^{-1}$ ) foram superiores às das demais condutividades avaliadas. Conclui-se que a posição do cacho floral na planta não afeta os componentes do rendimento número e peso médio do fruto, exercendo pouca influência sobre a produção de frutos por cacho do tomateiro cereja. Já, a variação da concentração iônica da solução nutritiva (na faixa entre $1,3 \mathrm{e} 2,8 \mathrm{dS} \mathrm{m}^{-1}$ ) não afeta o número de frutos colhidos por planta, mas uma $\mathrm{CE}$ superior a $2,3 \mathrm{dS} \mathrm{m}^{-1}$ provoca uma redução no peso médio do fruto e, consequentemente, na produção de frutos por planta.

Palavras-chave: Lycopersicon esculentum Mill, hidroponia, produção, NFT, técnica do filme de nutrientes.

\begin{abstract}
Yield of cherry tomatoes depending on the floral cluster and the concentration of nutrients in hydroponics

The components of yield (number and average weight of fruits per plant) of cherry tomatoes grown in hydroponic system were evaluated, in the summer-autumn 2008 season, noting the influence of the floral cluster position and the nutrients concentration on the solution. Four concentrations of ionic nutrient solution were studied (initial electrical conductivity (CE)): $1.3 ; 1.8 ; 2.3$ and $2.8 \mathrm{dS} \mathrm{m}^{-1}$. There was no significant interaction between the factors nutrients concentration of the nutrient solution and position of the floral cluster for all evaluated yield components. Regarding the effect of the position of the floral cluster, significant influence only occurred for the variables fruit setting index and fruit production. The ion concentration of the nutrient solution had significant effect on the following yield variables: fruit mean weight and production of fruits per plant. In terms of fruit mean weight, the CEs of 1.8 and $2.3 \mathrm{dS} \mathrm{m}^{-1}$ were higher than the $\mathrm{CE}$ of $2.8 \mathrm{dS} \mathrm{m}^{-1}$ and similar to the $\mathrm{CE}$ of $1.3 \mathrm{dS}$ $\mathrm{m}^{-1}$ resulting in $13.0 ; 12.5 ; 9.5$ and 10.9 grams per fruit, respectively. The average production of fruits of plants grown in nutrient solution with CE of $1.8 \mathrm{dS} \mathrm{m}^{-1}$ (296.5 $\mathrm{g}$ cluster $^{-1}$ and $2.1 \mathrm{~kg}$ plant $\left.^{-1}\right)$ were superior to the other evaluated conductivities. The position of the floral cluster in the plant does not affect the components of yield, number and mean weight of the fruit. Thus, the position of the floral cluster has little influence on the production of fruits per cluster of cherry tomatoes. The change in the ion concentration of nutrient solution (in the range from 1.3 to $2.8 \mathrm{dS} \mathrm{m}^{-1}$ ) does not affect the number of fruits per plant, but an CE higher than $2.3 \mathrm{dS} \mathrm{m}^{-1}$ reduces the fruit mean weight.
\end{abstract}

Keywords: Lycopersicon esculentum Mill, hydroponic system, production, NFT, nutrient film technique.

\section{(Recebido para publicação em 8 de julho de 2009; aceito em 21 de outubro de 2010) (Received on July 8, 2009; Accepted on October 21, 2010)}

$\mathrm{O}$ rendimento de uma hortaliça de fruto é determinado pela combinação de dois componentes: número e peso médio de frutos colhidos por planta, cuja associação resulta na produção por planta. O número de frutos produzido é uma conseqüência direta do índice de pegamento de frutos na planta. O peso médio de fruto é um relevante componente da produção, além de ser a melhor maneira de exprimir, indiretamente, o tamanho dos frutos.

$\mathrm{O}$ rendimento de frutos do tomateiro é determinado pelo balanço entre crescimento vegetativo e reprodutivo para um determinado fornecimento de assimilados (Ho, 1996). A alocação dos assimilados para os frutos e, consequentemente, o rendimento da planta do tomateiro, dependem principalmente do número de frutos existentes na planta (Heuvelink, 1996).

Em caso de hortaliças cuja inflorescência apresenta-se na forma de cacho floral, como o tomate, a potência de dreno do fruto e, consequentemente, seu tamanho, são influenciados pela posição do fruto no cacho floral e pela posição do cacho floral no caule. Os primeiros frutos exercem efeito dominante sobre os que aparecem posteriormente. Aqueles tendem a monopolizar os assimilados disponíveis, de forma que as flores e os frutos recém formados muitas vezes deixam de crescer e abortam. Os frutos distais têm uma menor potência dreno que os frutos proximais dentro de um mesmo cacho floral. Os frutos distais também são submetidos a uma dupla competição, dentro e entre cachos florais (Bertin, 1995). Além disso, o crescimento dos frutos a partir do $5^{\circ}$ cacho floral 
pode diminuir, pois a competição por assimilados é muito alta nesta fase. Contudo, os cachos florais subseqüentes, a partir do sétimo, voltam a crescer normalmente, quando os primeiros alcançam a maturação e diminui a competição por assimilados, o que caracterizaria um crescimento diferenciado entre frutos de distintos cachos florais de uma mesma planta (Bertin \& Gary, 1992). Por sua vez, o número de frutos que cresce em uma planta mantém uma forte dependência das intensidades de formação, aborto, crescimento, desenvolvimento e colheita.

As informações reportadas se referem a grupos de hortaliças de frutos de tamanhos médio e grande. Porém, quanto aos mini-tomates, poucas informações estão disponíveis e não se tem conhecimento se as relações mencionadas se dão de maneira similar ao anteriormente exposto. Devido à pequena potência individual de dreno de cada fruto do tomate cereja, tais relações podem ocorrer de maneira diferenciada.

Existe diversidade entre as respostas obtidas em diferentes estudos sobre os efeitos da variação da concentração iônica da solução nutritiva sobre a qualidade e os componentes do rendimento de hortaliças de fruto em cultivo hidropônico. Kawakami et al. (2007), avaliando a produtividade da cultura do tomate cereja em função de diferentes condutividades elétricas (CE) da solução nutritiva no ciclo outono-inverno, observaram que na medida em que se aumentou a CE (desde 1,3 até 3,8 dS $\mathrm{m}^{-1}$ ) ocorreu uma diminuição na massa média dos frutos. Porém, não suficiente para promover diferenças significativas entre as CE estudadas no que se refere ao número de frutos por planta e produtividade. Claussen (2002), trabalhando com tomate, observou que variando de 1 a 5 vezes a concentração da solução nutritiva original não houve alteração tanto no crescimento vegetativo quanto no reprodutivo da planta. Do mesmo modo, Genúncio et al. (2006) observaram que diluições da solução original a $50 \%\left(1,4 \mathrm{dS} \mathrm{m}^{-1}\right)$ não influenciaram o número total de frutos por planta e o acúmulo de massa de cultivares de tomateiro saladinha, cultivadas no período primavera-verão. Trabalhos de Torres et al. (2004) e Siddiqi et al. (1998) mostram ser possível reduzir a concentração da solução nutritiva a níveis abaixo da força iônica original das soluções comumente usadas em cultivos hidropônicos em sistemas recirculantes de tomate, sem que se incorra em riscos de perda da produtividade.

A concentração ótima de nutrientes de uma solução nutritiva está diretamente relacionada com a demanda evaporativa da atmosfera (Stanghellini, 1987). Portanto, a definição da concentração a ser utilizada deve ser objeto de estudo, tendo em vista as diferenças genotípicas, ambientais e as demandas associadas às diferentes fases do desenvolvimento de uma cultura (Martinez, 2002).

O objetivo do presente trabalho foi gerar conhecimento sobre os componentes do rendimento e a produção do tomate cereja, cultivado em sistema hidropônico, observando a influência da posição do cacho floral e da concentração iônica da solução nutritiva.

\section{MATERIAL E MÉTODOS}

O experimento foi realizado no Campus da Universidade Federal de Pelotas $\left(31^{\circ} 52^{\prime} \mathrm{S}, 52^{\circ} 21^{\prime} \mathrm{W}\right.$, altitude 13 $\mathrm{m})$, de $03 / 03$ a 30/05/08. O ensaio foi conduzido em uma estufa modelo "Arco Pampeana", disposta no sentido NorteSul, revestida com filme plástico PEBD (150 $\mu \mathrm{m}$ de espessura), compreendendo uma área de $210 \mathrm{~m}^{2}(10$ x $21 \mathrm{~m})$, com o solo coberto com filme de polietileno dupla face (branco/preto).

Foram utilizadas sementes de tomate Cereja Vermelho, linha Blue Line da Topseed Garden ${ }^{\circledR}$. No dia 28/01/08, realizou-se a semeadura em bandejas plásticas, preenchidas com substrato de vermiculita expandida. Aos 16 dias após a semeadura (DAS), quando as plantas emitiram a terceira folha definitiva, as mesmas foram repicadas para cubos de espuma fenólica de 2,5 x 2,5 x $3 \mathrm{~cm}$, dispostos em sistema flutuante para fertirrigação. Na fase de produção de mudas, utilizou-se a mesma solução nutritiva recomendada pela "Japan Horticultural Experimental Station" na concentração de 50\%, mantendo-se uma lâmina de solução de aproximadamente $1,0 \mathrm{~cm}$ de altura. O transplante foi rea- lizado quando as mudas apresentavam em torno de 7 folhas definitivas, aos 35 DAS (03/03/08).

A técnica de cultivo hidropônico utilizada foi a NFT (técnica da lâmina de nutrientes, Cooper, 1973). O sistema foi constituído por 10 canais de madeira $(7,5 \mathrm{~m} \times 35 \mathrm{~cm})$, com declividade de $2 \%$, dispostos em 5 linhas duplas, com distância interna de $50 \mathrm{~cm}$ e passeio de 1,2 m. Internamente, os canais foram revestidos com filme de polietileno dupla face preto-branco, de maneira a formar canais impermeáveis. Um tanque de armazenamento da solução nutritiva se encontrava enterrado próximo à extremidade de cota menor, ao final de cada linha dupla de canais. Um conjunto moto-bomba de 1/4 HP fixado em cada tanque impulsionava a solução nutritiva para a extremidade de maior cota dos canais através de um cano de PVC de $25 \mathrm{~mm}$, na vazão de $12 \mathrm{~L} \mathrm{~min}^{-1}$. A partir desse ponto, devido à declividade, a solução nutritiva percorria a base dos canais de cultivo, formando uma lâmina fina, e após passar pelas raízes, retornava para o reservatório, formando um sistema fechado.

A concentração iônica da solução nutritiva foi mantida em quatro níveis: solução nutritiva padrão (100\% da concentração de macronutrientes); soluções nutritivas com reduções de $25 \%$ e $50 \%$ da concentração de nutrientes em relação à solução padrão; e solução nutritiva com aumento de $25 \%$ da concentração de nutrientes em relação à solução padrão. Os micronutrientes foram mantidos na concentração padrão em todos os tratamentos estudados. A solução nutritiva padrão utilizada foi a recomendada pela "Japan Horticultural Experimental Station” (Peil et al., 1994), com a seguinte composição expressa em mmol L-1 : 16,0 de $\mathrm{NO}_{3} ; 1,3$ de $\mathrm{H}_{2} \mathrm{PO}_{4}$; 2,0 de $\mathrm{SO}_{4} ; 1,3$ de $\mathrm{NH}_{4} ; 8,0$ de $\mathrm{K} ; 4,0$ de $\mathrm{Ca}$; 2,0 de $\mathrm{Mg}$; e, em mg L-1, 3,0 de Fe; 0,5 de Mn; 0,05 de Zn; 0,15 de B; 0,02 de $\mathrm{Cu}$ e 0,01 de Mo. Partindo-se da solução nutritiva padrão preparou-se as soluções nutritivas dos tratamentos, utilizando-se água da chuva $(\mathrm{CE}=0,0$ $\mathrm{dS} \mathrm{m} \mathrm{m}^{-1}$ ).

Após o preparo, a condutividade elétrica de cada solução foi medida com eletrocondutivímetro digital, marca 
Instrutherm CD-840, estabelecendo-se os quatro níveis de concentração iônica estudados: 1,$3 ; 1,8 ; 2,3$ e $2,8 \mathrm{dS} \mathrm{m}^{-1}$, respectivamente para as soluções nutritivas com reduções de $25 \%$ e $50 \%$ da concentração de nutrientes; solução nutritiva padrão e solução nutritiva com aumento de $25 \%$ da concentração de nutrientes.

A solução nutritiva foi monitorada diariamente através das medidas de condutividade elétrica (empregando-se um eletrocondutivímetro digital) e de $\mathrm{pH}$ (empregando-se um pHmetro digital), sendo este mantido entre 5,8 e 6,3, através da adição de solução de correção à base de ácido sulfúrico $\left(\mathrm{H}_{2} \mathrm{SO}_{4} 1 \mathrm{~N}\right) \mathrm{ou}$ hidróxido de sódio $(\mathrm{NaOH} 1 \mathrm{~N})$.

A reposição de nutrientes ou de água foi realizada através da adição de soluções estoque concentradas ou de água da chuva estocada, quando o valor da condutividade elétrica sofreu, respectivamente, uma diminuição ou um aumento, da ordem de $10 \%$, de maneira similar à empregada anteriormente por Duarte et al. (2008), Montezano (2003, 2007) e Strassburger (2007).

O fornecimento da solução nutritiva foi realizado de forma intermitente, com $15 \mathrm{~min}$ de fornecimento e $30 \mathrm{~min}$ de intervalo entre 8:00 e 10:00 h e entre 16:30 e 19:00 h. No horário de 10:00 a 16:30 h, o tempo de fornecimento foi estendido para $30 \mathrm{~min}$. Durante a noite, realizava-se apenas uma irrigação de 15 min, à 1:00 h.

As plantas foram espaçadas a $35 \mathrm{~cm}$ nos canais de cultivo, o que resultou em uma densidade de 3,36 plantas $\mathrm{m}^{-2}$, totalizando 21 plantas por canal. As plantas foram conduzidas verticalmente, em haste única, com uso de fita de ráfia presa a uma linha de arame disposta cerca de 3,0 $\mathrm{m}$ acima da linha de cultivo.

$\mathrm{O}$ início do florescimento ocorreu aos 10 dias após o transplante (DAT), sendo feita, a partir de então, a contagem das flores emitidas por cacho floral. $\mathrm{O}$ período de colheita dos frutos, que já haviam iniciado a mudança de coloração de verde para vermelho, iniciou-se aos 45 DAT e teve duração de 43 dias. Em cada colheita, os frutos de cinco plantas por tratamento foram contados e pesados individualmente por cacho (do $1^{\mathrm{o}}$ ao $7^{\circ}$ cacho emitido), e com base nesses dados, determinaram-se o índice de pegamento dos frutos e os componentes do rendimento: número de frutos, produção de frutos e peso médio do fruto, tanto por cacho floral quanto por planta.

O delineamento experimental adotado foi inteiramente casualizado em esquema bifatorial: posição do cacho floral (fator qualitativo com sete níveis) e concentração iônica da solução nutritiva (fator quantitativo com quatro níveis), totalizando 28 tratamentos experimentais, com cinco repetições.

Os dados obtidos foram submetidos à análise da variância e testes de hipóteses através da análise bifatorial. Interpretaram-se os níveis do fator qualitativo através da comparação de médias pelo teste de Tukey a 5\% de probabilidade e os do fator quantitativo por meio da análise de regressão, sendo obtidos a equação estimada e os pontos de máxima eficiência técnica.

\section{RESULTADOS E DISCUSSÃO}

A partir da análise de variância observou-se que não houve interação significativa entre os fatores posição do cacho floral e concentração iônica da solução nutritiva para as quatro variáveis analisadas, o que permitiu a análise e apreciação dos resultados sobre os efeitos principais dos fatores separadamente.

Com relação ao efeito da posição do cacho floral, verificou-se que somente para a variável índice de pegamento de frutos, este apresentou influência significativa nas respostas observadas. Observou-se que o quinto cacho apresentou o maior índice de pegamento de frutos, $88,8 \%$, diferindo significativamente somente do sétimo cacho, que apresentou $70,9 \%$ de pegamento de frutos. Entretanto, esta diferença não afetou significativamente os componentes do rendimento (número, peso médio e produção de frutos por cacho), devido ao fato do sétimo cacho ter apresentado número superior de flores formadas $(21,3 \%$ a mais que o quinto cacho). O quarto cacho apresentou uma produção média de $261,7 \mathrm{~g}$, diferindo significativamente do sexto cacho, que apresentou média de $168,6 \mathrm{~g}$, sendo esta a única diferença significativa observada em relação a esta variável.

Independentemente da posição do cacho floral, os resultados relativos aos demais componentes do rendimento foram equivalentes às médias observadas: número de 19,5 frutos, peso médio do fruto de $11,5 \mathrm{~g}$ e produção de 204,1 g cacho ${ }^{-1}$. Tais resultados podem ser justificados pelo fato de que plantas com números semelhantes de fruto apresentam uma alocação similar de assimilados para os frutos (De Konning, 1994). A homogeneidade de respostas em função da posição do cacho floral observada para o tomate cereja neste trabalho difere do comportamento diferenciado entre cachos observado por Bertin \& Gary (1992) em tomate do tipo salada. O pequeno tamanho dos frutos do tomate cereja indica que estes são drenos muito menos potentes do que os maiores frutos do tomate salada, o que leva a crer que a competição por assimilados entre cachos não se estabelece ao ponto de um cacho prejudicar os componentes do rendimento do outro, diferentemente do observado para frutos de tomates maiores.

O peso médio dos frutos e a produção de frutos por planta reduziram com o aumento da concentração iônica da solução nutritiva (Fig. 1). Em relação ao número de frutos, não se observou influência da concentração iônica da solução nutritiva nas respostas apresentadas (Fig. 1a). Independentemente da concentração iônica, os resultados relativos a este componente do rendimento foram equivalentes à média obtida de 133,7 frutos. Para a variável índice de pegamento de frutos, também, não se observou influência significativa da concentração da solução nutritiva. As quatro concentrações de nutrientes avaliadas apresentaram média de 81,3\% de pegamento de frutos.

Em termos de peso médio de frutos (Fig. 1b), as CEs de 1,8 e 2,3 $\mathrm{dS} \mathrm{m}^{-1}$ mostraram-se superiores à $\mathrm{CE}$ de 2,8 $\mathrm{dS} \mathrm{m}^{-1}$ e similares à $\mathrm{CE}$ de $1,3 \mathrm{dS} \mathrm{m}^{-1}$, obtendo-se 13,$0 ; 12,5 ; 9,5$ e $10,9 \mathrm{~g}$ por fruto, respectivamente. A máxima eficiência técnica (MET) da solução nutritiva foi alcançada com $\mathrm{CE}$ de $1,9 \mathrm{dS}$ $\mathrm{m}^{-1}$ para a qual obtém-se peso médio de fruto de 13,3 g. Resultados similares foram obtidos por Kawakami et al. (2007) 


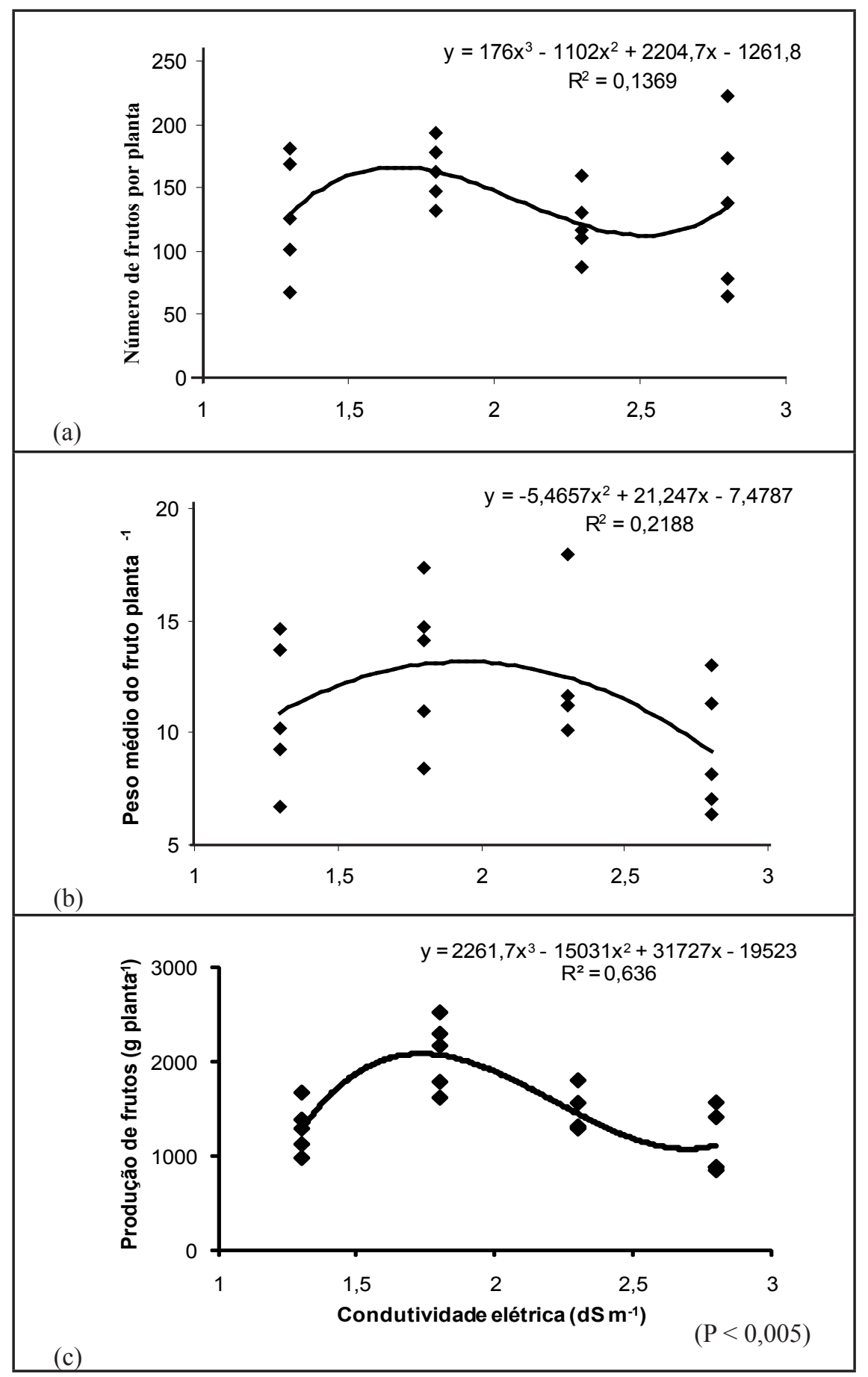

Figura 1. Efeito da concentração iônica da solução nutritiva (expressa através da condutividade elétrica) sobre os componentes do rendimento do tomateiro cereja em sistema hidropônico (ion concentration of the nutrient solution (in terms of electrical conductivity) effects on the yield components of cherry tomato grown in hydroponic system). Pelotas, UFPel, 2008.

e Campos et al. (2006) que avaliando o efeito da $\mathrm{CE}$ da solução nutritiva no cultivo de tomate cereja e tomate industrial, respectivamente, durante o ciclo outono-inverno, observaram que o aumento da CE da solução nutritiva causou diminuição na massa média dos frutos. Comportamento similar foi en- vadas na solução nutritiva com $\mathrm{CE}$ de

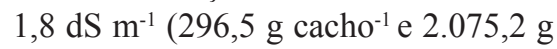
planta $^{-1}$ ) foram superiores às das demais condutividades avaliadas. $\mathrm{O}$ ponto de máxima produção foi alcançado com a CE de 1,7 dS m${ }^{-1}$, perfazendo uma produção de $297,9 \mathrm{~g} \mathrm{cacho}^{-1}$ e $2.085 \mathrm{~g}$ planta $^{-1}$. Esta resposta difere da observada por Kawakami et al. (2007) que não observaram diferenças significativas entre as produtividades de tomateiros cereja cultivados em soluções nutritivas com $\mathrm{CE}$ entre 1,3 e 3,8 dS m ${ }^{-1}$. O número de frutos por planta não foi afetado significativamente pela $\mathrm{CE}$ da solução nutritiva ao contrário do peso médio do fruto, o que implica em dizer que a maior produção por planta obtida para a $\mathrm{CE}$ de $1,8 \mathrm{dS} \mathrm{m}^{-1}$ deveu-se, principalmente, ao maior peso médio dos frutos e, depois, a combinação deste com o maior valor absoluto do número de frutos colhidos neste tratamento.

Os resultados em relação à concentração iônica também diferem dos obtidos por Genúncio et al. (2006) e Torres et al. (2004) que, ao cultivarem tomate do tipo salada sob diferentes concentrações iônicas de solução nutritiva (de 1,4 até 2,9 $\mathrm{dS} \mathrm{m}^{-1}$ ) em sistema hidropônico, durante o ciclo primavera-verão, verificaram que as mesmas variáveis de rendimento não diferiram nos tratamentos estudados. As respostas observadas por estes autores indicam, possivelmente, que as soluções nutritivas utilizadas foram desenvolvidas para suprir condições de alta exigência nutricional da cultura do tomateiro, podendo o tomate cereja então se desenvolver em soluções menos concentradas. Entretanto, Andriolo et al. (2003) observaram que a produtividade de frutos de tomateiro decresceu com o aumento da CE da solução nutritiva, porém, este efeito negativo somente foi observado com valores superiores a 4,9 dS m $\mathrm{d}^{-1}$, enquanto que no presente trabalho tal fato ocorreu para a CE de 2,3 $\mathrm{dS} \mathrm{m}^{-1}$, nos levando a acreditar em uma maior sensibilidade do tomate cereja à alta salinidade. Li (2000), também verificou um decréscimo na produtividade de frutos de tomateiro $c v$ Chaser na medida em que se aumentou a $\mathrm{CE}$ da solução nutritiva (de 2,3 até $6,8 \mathrm{dS} \mathrm{m}^{-1}$ ), corroborando com os resultados obtidos por Sonneveld (1988) de que o limite da 


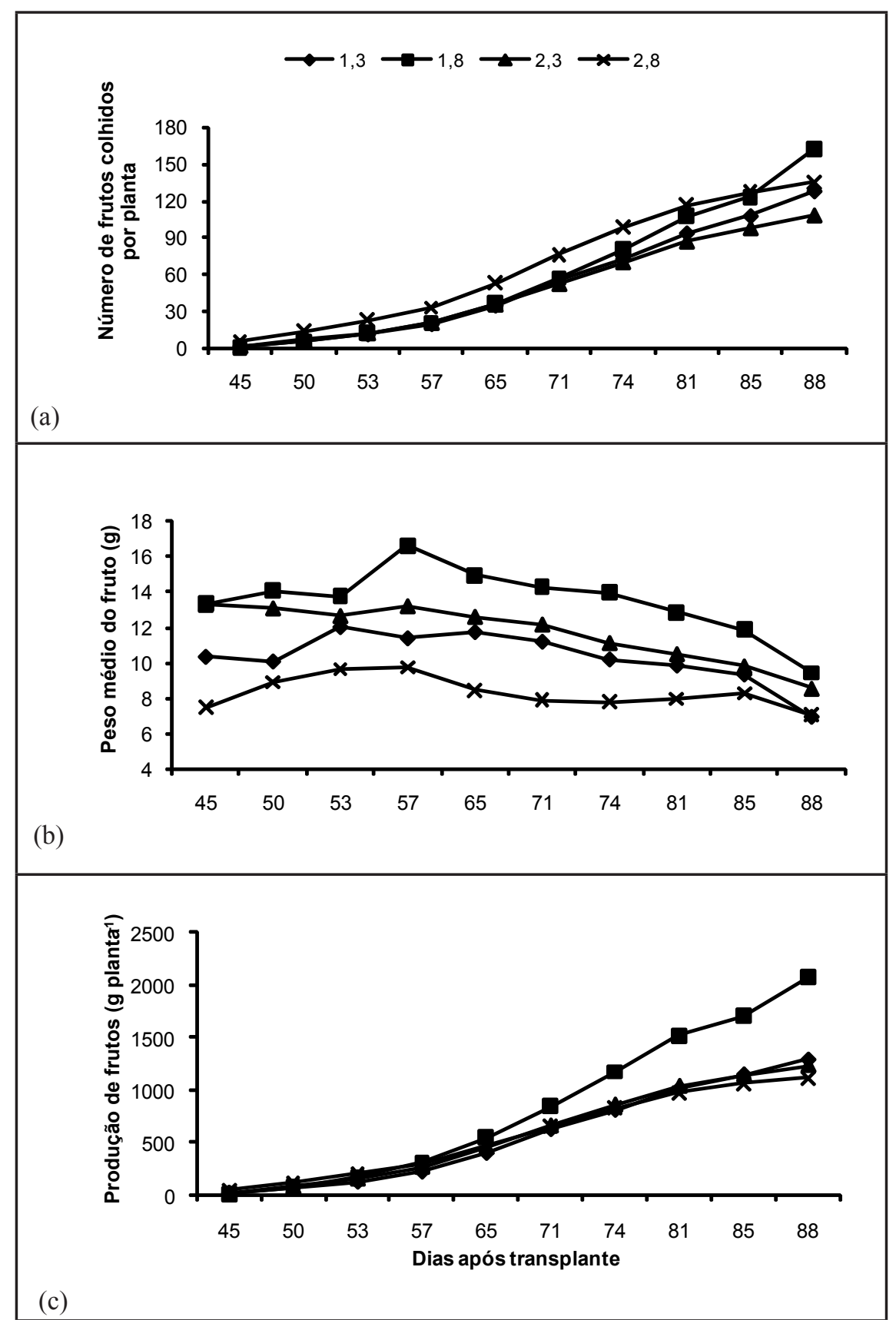

Figura 2. Evolução dos valores dos componentes de rendimento do tomate cereja em função da concentração iônica da solução nutritiva (expressa pela condutividade elétrica, CE, em dS $\mathrm{m}^{-1}$ ) ao longo do ciclo de cultivo, em sistema hidropônico (evaluation of yield components of cherry tomato depending on the ion concentration of the nutrient solution (in terms of electrical conductivity, $\mathrm{CE}$, in $\mathrm{dS} \mathrm{m}^{-1}$ ) during the cultivation cycle, in hydroponic system). Pelotas, UFPel, 2008.

CE para a cultura do tomateiro é de 2,5 $\mathrm{dS} \mathrm{m}^{-1}$, valor este similar ao observado na presente pesquisa.

Os baixos coeficientes de determinação $\left(\mathrm{R}^{2}\right)$ obtidos ao verificar-se o efeito da concentração iônica da solução nutritiva sobre os componentes do rendimento do tomateiro cereja, podem ser justificados pela alta variação natural destas respostas. Para a variável peso (número, peso médio e produção de frutos), indica que houve tendência de melhor resposta da planta à concentração iônica intermediária de $1,8 \mathrm{dS} \mathrm{m}^{-1}$. Tais resultados podem ser explicados pelo efeito da concentração dos nutrientes no meio radicular. Em baixas concentrações iônicas há baixa disponibilidade de nutrientes dissolvidos na água, propiciando respostas inferiores ao potencial produtivo da cultura, como o observado na $\mathrm{CE}$ de 1,3 $\mathrm{dS} \mathrm{m} \mathrm{m}^{-1}$. Em contrapartida, em concentrações elevadas, menor é a diferença entre os potenciais hídricos da solução nutritiva e do sistema radicular, dificultando a absorção de água, podendo também dificultar a própria absorção mineral (Andriolo, 1999), o que pode levar à diminuição do peso médio do fruto e, consequentemente, da produção de frutos. Segundo Cuartero \& Muñoz (1999), sob salinidade moderada a redução no rendimento do tomateiro devese, principalmente, à redução da massa média dos frutos, concordando com os resultados obtidos neste trabalho.

Uma das formas de avaliar-se a produtividade das culturas é considerando seu ciclo. Em tomateiros do tipo cereja, a avaliação da produção, considerando o tempo, permite uma melhor visualização do cultivo. Na figura 2, encontra-se a distribuição dos valores de número de frutos acumulados, peso médio e produção acumulada de frutos, ao longo do ciclo de cultivo em função da concentração iônica da solução.

Com relação à variável número de frutos, percebeu-se a mesma tendência comportamental ao longo do ciclo de cultivo entre os quatros tratamentos avaliados (Fig. 2a). Ao final do ciclo, o número de frutos colhidos por planta (média de 136,8 frutos) foi similar ao observado por Bavuso Neto et al. (2006) que obtiveram resultados variando entre 92 e 176 frutos por planta. Números superiores, de até 328 frutos por planta, foram observados por Postali et al. (2004) no híbrido comercial Pepe. A colheita de maior número de frutos maduros foi observada aos 81 DAT, na qual se coletou 20,7 frutos por planta.

Na colheita realizada aos 57 DAT, verificaram-se os maiores valores de peso médio do fruto para a $\mathrm{CE}$ de $1,8 \mathrm{dS}$ $\mathrm{m}^{-1}$ (Fig. 2b). Ao término do cultivo, o 
peso médio do fruto obtido nas soluções nutritivas de CE de 1,$3 ; 1,8$ e $2,3 \mathrm{dS} \mathrm{m}^{-1}$ enquadra-se ao padrão de peso do grupo cereja, de 10 a 30 g (Diez-Niclos, 1995) e, de acordo com a classificação proposta por Fernandes et al. (2007), ajusta-se a classe de frutos médios (entre 10 e 15 g). Já o peso médio do fruto obtido na $\mathrm{CE}$ de $2,8 \mathrm{dS} \mathrm{m}^{-1}$, enquadra-se na classe de frutos pequenos (entre 5 e $10 \mathrm{~g}$ ). Tais resultados assemelham-se aos encontrados por Bavuso Neto et al. (2006) e são superiores aos observados por Postali et al. (2004), em cultivo hidropônico no Brasil.

A produção total de frutos atingiu, aos 88 DAT, 1,3; 21; 1,5 e 1,1 kg planta ${ }^{-1}$, referentes às CEs de 1,$3 ; 1,8 ; 2,3$ e 2,8 $\mathrm{dS} \mathrm{m}^{-1}$, respectivamente (Fig. 2c). Tais produções no espaçamento adotado correspondem, respectivamente, a rendimentos de 43,3, 69,7, 48,8 e 37,5 t $\mathrm{ha}^{-1}$ ou 492, 792, 554 e $426 \mathrm{~kg} \mathrm{ha}^{-1} \mathrm{dia}^{-1}$, distribuídos em dez colheitas de frutos maduros. As produções foram mais concentradas durante dezesseis dias do ciclo, entre a quinta (65 DAT) e a oitava colheita (81 DAT). A maior produção por planta observada neste trabalho foi similar às obtidas por Postali et al. (2004) com o híbrido Super Sweet (2,1 $\left.\mathrm{kg} \mathrm{planta}^{-1}\right)$ e à mínima obtida por Bavuso Neto et al. (2006) (1,9 kg planta-1) em estudo com híbridos em sistema hidropônico, entretanto, foi inferior à observada pelo primeiro autor com o híbrido Pepe (3,0 kg planta-1).

Através dos resultados obtidos neste trabalho conclui-se que a posição do cacho floral na planta não afeta os componentes do rendimento número e peso médio do fruto, exercendo pouca influência sobre a produção de frutos por cacho do tomateiro cereja. A variação da concentração iônica da solução nutritiva (na faixa entre 1,3 e 2,8 $\mathrm{dS} \mathrm{m}^{-1}$ ) não afeta o número de frutos colhidos por planta, mas uma CE superior a 2,3 $\mathrm{dS} \mathrm{m}^{-1}$ provoca uma redução no peso médio do fruto e, consequentemente, na produção de frutos por planta.

Os dados observados neste trabalho levam a considerar que deve-se realizar, nas condições estudadas, uma redução de $25 \%$ da concentração iônica da solução nutritiva padrão (i.e., empregar a solução com CE de 1,8 dS m-1), obtendo-se desta forma uma elevação na produção final da cultura do tomate cereja.

\section{AGRADECIMENTOS}

Os autores Marcelo Q Rocha e Clarissa M Cogo agradecem a bolsa FAPEAM e CNPq, respectivamente.

\section{REFERÊNCIAS}

ANDRIOLO JL. 1999. Fisiologia das culturas protegidas. Santa Maria: Ed. UFSM. 142 p.

ANDRIOLO JL; WITTER M; DAL ROSS T; GODÓI RS. 2003. Crescimento e desenvolvimento do tomateiro cultivado em substrato com reutilização da solução nutritiva drenada. Horticultura Brasileira 21: 485-489.

BAVUSO NETO P; SILVA EC; MARQUES DJ; MACIEL GM. 2006. Desempenho de genótipos e cultivares híbridas comerciais de tomateiro do grupo cereja em sistema de cultivo hidropônico NFT. Horticultura Brasileira 24: 277-280.

BERTIN N. 1995. Competition for assimilates and fruit position affect fruit set in indeterminate greenhouse tomato. Annais of Botany 75: 55-65.

BERTIN N; GARY C. 1992. Tomato fruit set and competition for assimilates, during the early production period. Acta Horticulturae 303: 121-126.

CAMPOS CAB; FERNANDES PD; GHEYI HR; BLANCO FF; GONÇALVES CB; CAMPOS SAF. 2006. Yield and fruit quality of industrial tomato under saline irrigation. Science Agricultural 63: 146-152.

CLAUSSEN W. 2002. Growth, water use efficiency, and proline content of hydroponically grown tomato plants as affected by nitrogen source and nutrient concentration. Plant and Soil 00: $1-11$.

COOPER AJ. 1973. Rapid crop turn-round is possible with experimental nutrient film technique. Grower 79: 1048-1052.

CUARTERO J; MUÑOZ RF. 1999. Tomato and salinity. Scientia Horticulturae 78: 83-125.

DE KONNING ANM. 1994. Development and dry matter distribution in glasshouse tomato: a quantitative approach. Wageningen: Wageningen Agricultural University. 240p. (Tese doutorado).

DIEZ NICLOS J. 1995. Tipos varietables. In: NUEZ F (coord). El cultivo del tomate. Madrid: Mundi Prensa. p. 93-129.

DUARTE TS; PEIL RMN; BACCHIS S; STRASSBURGER AS. 2008. Efeito da carga de frutos e concentrações salinas no crescimento do meloeiro. Horticultura Brasileira 26: 346-351.

FERNANDES C; CORÁ JE; BRAZ LT. 2007. Classificação de tomate-cereja em função do tamanho e peso dos frutos. Horticultura Brasileira 25: 275-278.

GENÚNCIO GC; MAJEROWICZ N; ZONTA E; SANTOS AM; GRACIA D; AHMED CRM; SILVA MG. 2006. Crescimento e produtividade do tomateiro em cultivo hidropônico NFT em função da concentração iônica da solução nutritiva. Horticultura Brasileira 24: 175-179.
GUALBERTO G; BRAZ LT; BANZATTO DA. 2002. Produtividade, adaptabilidade e estabilidade fenotípica de cultivares de tomateiro sob diferentes condições de ambiente. Pesquisa Agropecuária Brasileira 37: 81-88.

HEUVELINK E. 1996. Tomato growth and yield: quantitative analysis and synthesis. Wageningen: Wageningen Agricultural University. 326 p. (Tese doutorado).

HO LC. 1996. Tomato. In: ZAMSTRI E; SCHAFFER AA. Photoassimilate distribution in plants and crops. New York: Marcel Dekker. $905 \mathrm{p}$.

KAWAKAMI FPC; ARAUJO JAC; IUNCK AV; FACTOR TL; CORTEZ GE. 2007. Manejo da fertirrigação em função da condutividade elétrica da solução nutritiva drenada no cultivo de tomate cereja sob ambiente protegido. Horticultura Brasileira 25: Suplementoresumo.

LI YL. 2000. Analysis of greenhouse tomato production in relation to salinity and shoot environment. Wageningen: Wageningen Agricultural University. 96 p. (Tese doutorado).

MARTINEZ HEP. 2002. O uso do cultivo hidropônico de plantas em pesquisa. Viçosa: UFV. $61 \mathrm{p}$.

MONTEZANO EM. 2003. Eficiência no uso da água e dos nutrientes e relações de contaminação de cultivos de alface em sistema hidropônico. Pelotas: UFPel, FAEM. 60 p. (Dissertação mestrado).

MONTEZANO EM. 2007. Sistemas de cultivo sem solo para a cultura do meloeiro. Pelotas: UFPel, FAEM. 141 p. (Tese doutorado).

PEIL RMN; BOONYAPORN S; SAKUMA H. 1994. Effect of different media on the growth of tomato in soilless culture. Report on Experiments in Vegetable Crops Production 53: 61-65.

POSTALI GB; SILVA EC; MACIEL GM. 2004. Produção de híbridos comerciais de tomateiro do grupo cereja cultivados no sistema hidropônico com diferentes números de hastes. Horticultura Brasileira 22: Suplemento 2.

SIDDIQI MV; KRONZUCKER HJ; BRITTO DT; GLASS DM. 1998. Growth of a tomato crop at reduced nutrient concentrations as a strategy to limit eutrophication. Journal Plant Nutrition 21: $1879-1895$

SONNEVELD C. 1988. The salt tolerance of greenhouse crops. Netherlands Journal of Agricultural Science 36: 63-73. STANGHELLINI C. 1987. Transpiration of greenhouse crops. An aid to climate management. Wageningen: Wageningen Agricultural University. 150 p. (Tese doutorado).

STRASSBURGER AS. 2007. Cultivo da abobrinha italiana em substrato de casca de arroz em ambiente protegido com solução nutritiva recirculante. Pelotas: UFPel, FAEM. 118 p. (Dissertação mestrado).

TORRES OGV; GARCIA PS; CASTILLO GAB; MENDONZA MNR; LÓPEZ CT; VILLAMS; SORIANO EC. 2004. Desarrollo y producción de tomate (Lycopersicon esculentum Mill.) con solución nutritiva específica para cada etapa fenológica. In: REUNIÃO BRASILEIRA DE FERTILIDADE DO SOLO E NUTRIÇÃO DE PLANTAS, FERTBIO, 26. Resumos... Lajes: (CD-ROM). 